\title{
Ignition Analysis of a Porous Energetic Material --- II. Ignition at a Closed Heated End
}

RTERVER

FER 1 ? 2900

STI

Alexander M. Telengator, Stephen B. Margolis, and Forman A. Williams

Prepared by

Sandia National Laboratories

Albuquerque, New Mexico 87185 ?and Livermore, California 94550

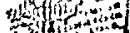

Sandia is a multiprogram laboratory operated by Sandia Corporation

a Lockheed Martin Company, for the United States Departmejt of .

Energy under Contract DE-AC04-94AL85000
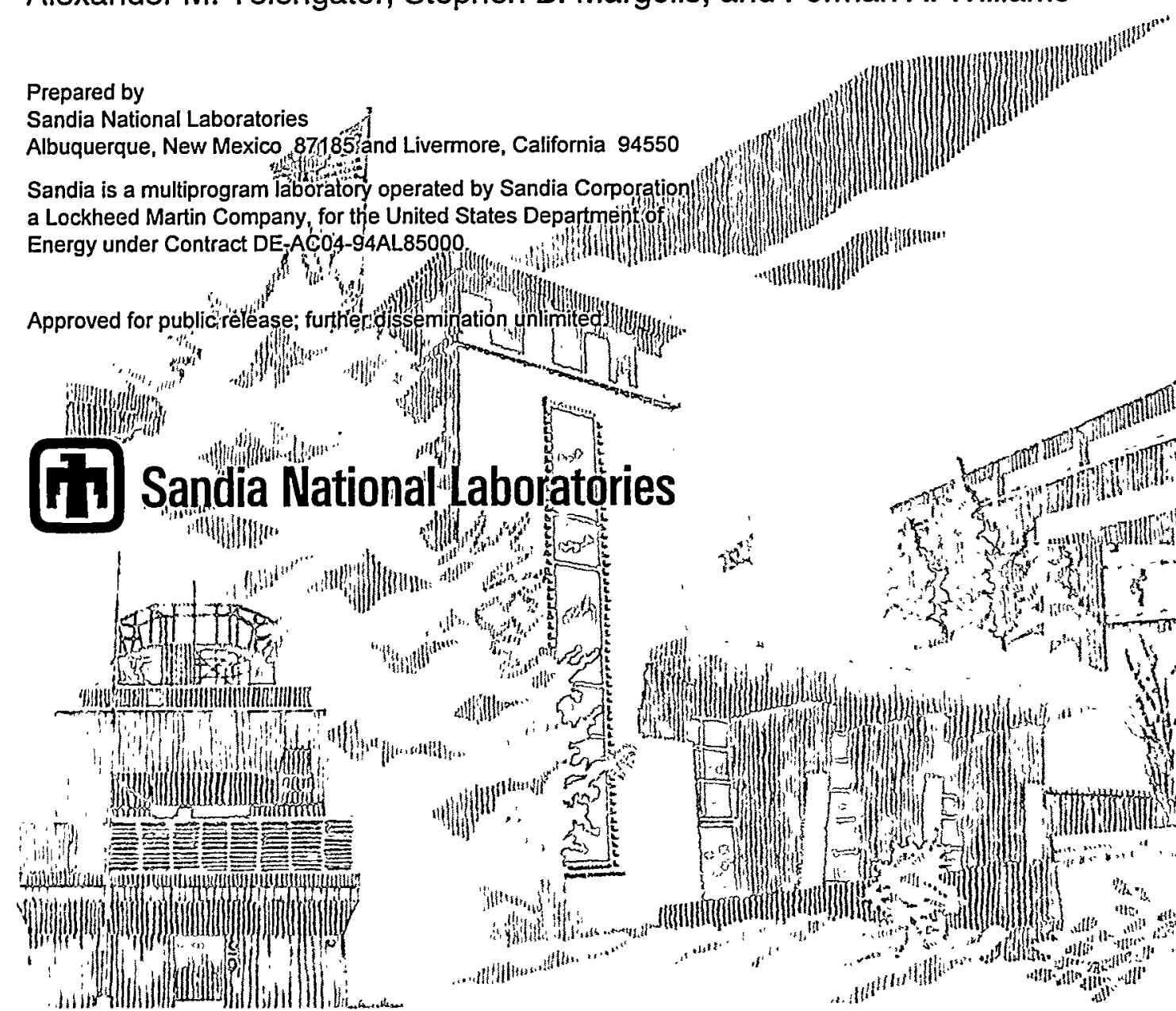

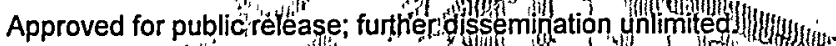
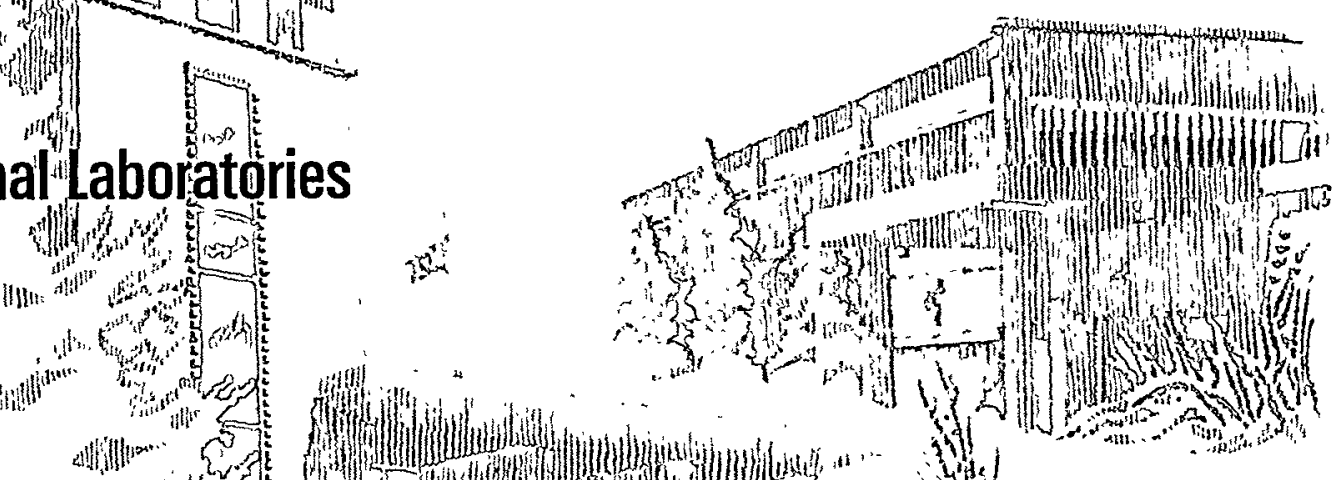

SF2900Q(8-81)
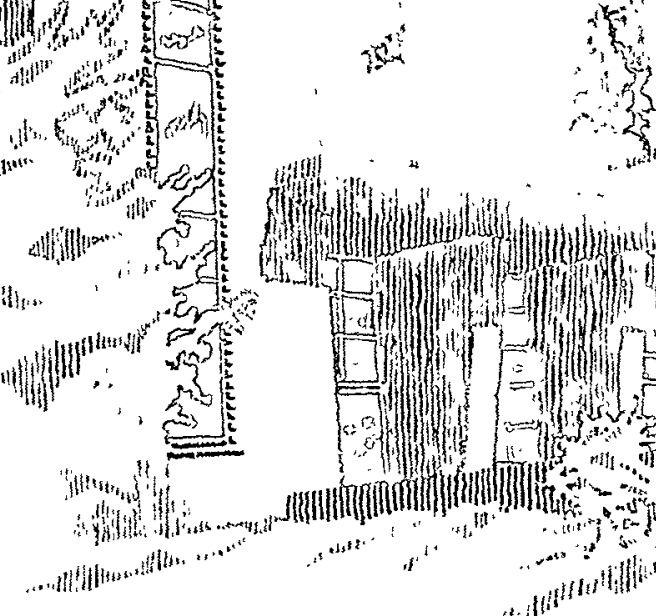
Issued by Sandia National Laboratories, operated for the United States Department of Energy by Sandia Corporation.

NOTICE: This report was prepared as an account of work sponsored by an agency of the United States Government. Neither the United States Government, nor any agency thereof, nor any of their employees, nor any of their contractors, subcontractors, or their employees, make any warranty, express or implied, or assume any legal liability or responsibility for the accuracy, completeness, or usefulness of any information, apparatus, product, or process disclosed, or represent that its use would not infringe privately owned rights. Reference herein to any specific commercial product, process, or service by trade name, trademark, manufacturer, or otherwise, does not necessarily constitute or imply its endorsement, recommendation, or favoring by the United States Government, any agency thereof, or any of their contractors or subcontractors. The views and opinions expressed herein do not necessarily state or reflect those of the United States Government, any agency thereof, or any of their contractors.

Printed in the United States of America. This report has been reproduced directly from the best available copy.

Available to $\mathrm{DOE}$ and $\mathrm{DOE}$ contractors from

Office of Scientific and Technical Information

P.O. Box 62

Oak Ridge, TN 37831

Prices available from (615) 576-8401, FTS 626-8401

Available to the public from

National Technical Information Service

U.S. Department of Commerce

5285 Port Royal Rd

Springfield, VA 22161

NTIS price codes

Printed copy: A03

Microfiche copy: A01

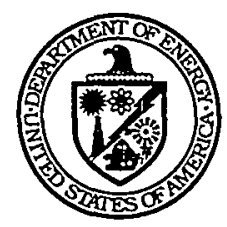




\section{DISCLAIMER}

Portions of this document may be illegible in electronic image products. Images are produced from the best available original document. 
SAND98-8655

Unlimited Release

Printed November 1998

\title{
IGNITION ANALYSIS OF A POROUS ENERGETIC MATERIAL - II. IGNITION AT A CLOSED HEATED END
}

\author{
Alexander M. Telengator ${ }^{\dagger}$, Stephen B. Margolis ${ }^{\ddagger}$ and Forman A. Williams ${ }^{\dagger}$ \\ †Department of Applied Mechanics and Engineering Sciences \\ University of California, San Diego \\ La Jolla, California 92093-0411 USA \\ $\ddagger_{\text {Combustion Research Facility }}$ \\ Sandia National Laboratories \\ Livermore, California 94551-0969 USA
}

\begin{abstract}
A continuation of an ignition analysis for porous energetic materials subjected to a constant energy flux is presented. In the first part (I), the analysis was developed for the case of an openend, semi-infinite material such that gas flow, generated by thermal expansion, flowed out of the porous solid, thereby removing energy from the system. In the present study, the case of a closed end is considered, and thus the thermally-induced gas flow is now directed into the solid. In these studies, an asymptotic perturbation analysis, based on the smallness of the gas-to-solid density ratio and the largeness of the activation energy, is utilized to describe the inert and transition stages leading to thermal runaway. In both cases it is found that the effects of porosity provide a leading-order reduction in the time to ignition relative to that for the nonporous problem, arising from the reduced amount of solid material that must be heated and the difference in thermal conductivities of the solid and gaseous phases. A correction to the leading-order ignition-delay time, however, is provided by the convective flow of gas through the solid, and the sign of this correction is shown to depend on the direction of the gas flow. Thus, gas flowing out of an open-end solid was previously shown to give a positive correction to the leading-order time to ignition. Here, however, it is demonstrated that when the flow of gas is directed into the porous solid, the relative transport effects associated with the gas flow serve to preheat the material, resulting in a negative correction and hence a decrease in the ignition-delay time.
\end{abstract}




\section{IGNITION ANALYSIS OF A POROUS·ENERGETIC MATERIAL - II. IGNITION AT A CLOSED HEATED END}

\section{Introduction}

As is well known, the ignition of combustible mixtures and materials from a nonreacted state is a fundamental topic in combustion theory. For many problems, such as the one considered here, it is characterized by a gradual increase in temperature through external heating, followed by a thermal explosion (i.e., a rapid temperature increase over a very short time) as exothermic reactions begin to occur. The time it takes for the latter to occur once such external heating has commenced is usually regarded as the time to ignition, or ignition-delay time, and is clearly a function of the precise problem being considered ( $c f$. Williams, 1985; Zel'dovich et al., 1985). In the present study, we consider the case of one type of spatially nonhomogeneous; two-phase problem in which heating occurs at the surface of a porous energetic material. In Part I (Telengator, Margolis and Williams, 1998, hereafter referred to as TMW), this surface was open in the sense that gas flow, generated by thermal expansion, was directed out of the solid. In the present study, we consider the case of a closed surface such that the thermally-induced gas flow is, by necessity, directed into the porous solid.

The ignition problems analyzed here and in Part I are generalizations of the one considered by Liñán and Williams (1971) (hereafter referred to as LW), which dealt with the ignition of a nonporous, semi-infinite, reactive solid by a constant heat flux at the surface; to address the ignition of a porous energetic material. In the latter problem, two-phase-flow effects begin to play a role in that gas flow, induced by thermal expansion, provides a means of convective transport of energy relative to the solid, as does any difference in physical properties of the two media with respect to diffusive transport. Since the ignition-delay time is inherently tied to the Arrhenius nature of the reaction-rate expressions, it is necessary to retain this feature of the problem in the analysis. Thus, in order to obtain approximate analytical estimates of the ignition-delay time and other aspects of the ignition process, we follow LW in applying activation-energy asymptotics to the two-phase problems considered in the present studies. Similar types of analyses have been applied to gaseous premixtures (cf. Kapila, 1981; Kassoy and Poland, 1980 \& 1981), where it has proven possible to continue the analysis past the point of ignition to describe the complete development of a propagating combustion wave. Problems dealing with the ignition of porous propellants and other energetic materials arise naturally from safety concerns associated with aged or damaged energetic materials that, as a consequence of either long-term storage or exposure to abnormal thermal environments; may have undergone some degree of decomposition and hence developed significant porosities with respect to their original pristine state. Although the heat flux at the surface may not be constant in these applications, the method employed here may be generalized 
to account for a time-varying energy flux (cf. Leiper and Roach, 1993).

The focus of the present study is a comparison of the two-phase-flow effects and ignitiontime results obtained for ignition at a closed surface of a porous energetic material with the previous results obtained in TMW for ignition at an open surface. In the damaged-propellant scenario, the open-surface problem corresponds to the ignition energy flux being applied where the casing has been thoroughly perforated, so that it provides no resistance to gas flow, while for the present closed-surface problem the casing at the heated surface remains intact. It will be shown, in particular, that opposite trends are predicted for the correction to the leading-order ignitiondelay time for the two problems, depending on which direction the gas flows relative to the heated surface. For the open-end problem, the flow of gas out the surface removes energy from the system, resulting in a corrective delay in the time to ignition, whereas the flow of gas into the solid for the closed-end problem effectively preheats the material and produces the opposite effect. It will be reasoned in the latter case that a nonzero convective gas velocity relative to the solid is established throughout the porous material during a very short initial stage of the ignition process.

\section{Mathematical Formulation}

Except for the difference in boundary conditions arising from the closed-end nature of the problem, as illustrated in Figure 1, the mathematical formulation of the problem is the same as in Part I. In particular, following the onset of external heating at the surface, we seek to determine the temporal history of the spatially varying solution up to the point of thermal explosion, or runaway, at some spatial location, signaling the onset of ignition. Based on our previous analysis (TMW) and that of LW, the following sequence of events is known to occur. Initially, the temperature is too low for chemical reaction to occur, and thus the first portion of the ignition process is characterized by an inert stage in which the solid is in a purely conductive state, though influenced by interphase heat transfer with the gaseous phase, which, because of its thermal expansion, has a relative motion with respect to the solid. The external heat flux eventually causes the temperature near the surface to reach a level at which exothermic chemical reactions can start to occur. Termed the transition stage in LW, this relatively short temporal regime is characterized by a thin surface region in which there is a reactive-diffusive balance, followed by a somewhat thicker transientdiffusive region in which solutions match with both the inner solutions in the reactive-diffusive zone and the inert solution in the far outer region where chemical effects are not yet felt. Thermal runaway then occurs in the inner region, marking the end of the transition stage and defining the time to ignition. Since the transition stage occurs on a short time scale relative to the inert stage of the heating, the leading-order time to ignition is essentially defined as the duration of the latter. For the small gas-to-solid density ratios considered here, the flow of gas relative to the solid will have a perturbative effect on the leading-order time to ignition, but the associated trend, along 
with other two-phase-flow aspects of the ignition process, will turn out to depend qualitatively on the direction of the gas flow.

The mathematical model used here and in TMW is based on the two-phase-flow formulation presented in several recent studies (Margolis and Williams, 1995a,b, 1996). We assume for simplicity a single-step exothermic reaction $R(c) \rightarrow P(g)$ in which the condensed (solid) material is converted directly to gaseous products, and consider the single-temperature limit of instantaneous interphase heat-transfer. Constant pressure is also assumed, based on the fact that gas velocities are small compared with the speed of sound and that even for the closed-end, semi-infinite problem considered here, gas can pass through the (realistically finite) sample and hence escape from that part of the physical domain of interest. Consequently, in terms of nondimensional quantities defined below, and under the assumption of large heat release $Q$ and a small gas-to-solid density ratio $\hat{r}$, the equation of state and the overall continuity and energy equations collapse to

$$
\begin{gathered}
\frac{\partial}{\partial t}\left(\alpha_{s} \rho_{g}\right)+\frac{\partial}{\partial \zeta}\left(\alpha_{s} \rho_{g} u_{g}\right)=0, \quad \dot{\rho_{g}}=1 / T \\
\frac{\partial}{\partial t}\left[\left(1-\alpha_{s}\right) T+\hat{r} \hat{b} \alpha_{s} u_{g}\right]+\hat{r} \hat{b} \frac{\partial}{\partial \zeta}\left(\alpha_{s} u_{g}\right)=\frac{\partial}{\partial \zeta}\left[\left(1-\alpha_{s}+\hat{l} \alpha_{s}\right) \frac{\partial T}{\partial \zeta}\right]+\hat{A}\left(1-\alpha_{s}\right) e^{-E / T}
\end{gathered}
$$

where the approximation of large heat release allows the neglect of reactant consumption and hence permits setting the porosity of the solid equal to its initial value $\alpha_{s}$. The various nondimensional quantities appearing above are defined in terms of their dimensional counterparts (denoted by tildes) according to

$$
\begin{gathered}
t=\frac{\tilde{\dot{q}}^{2} \tilde{t}}{\tilde{\lambda}_{s} \tilde{\rho}_{s} \tilde{c}_{s} \tilde{T}_{0}^{2}}, \quad \zeta=\frac{\tilde{\dot{q}} \tilde{\zeta}}{\tilde{\lambda}_{s} \tilde{T}_{0}}, \quad T=\frac{\tilde{T}}{\tilde{T}_{0}}, \quad \rho_{g}=\frac{\tilde{\rho}_{g}}{\tilde{\rho}_{g}^{0}}, \quad u_{g}=\frac{\tilde{\rho}_{s} \tilde{c}_{s} \tilde{T}_{0} \tilde{u}_{g}}{\tilde{\dot{q}}}, \quad Q=\frac{\tilde{Q}}{\tilde{c}_{s} \tilde{T}_{0}}, \\
E=\frac{\tilde{E}}{\tilde{R}^{\circ} \tilde{T}_{0}}, \quad A=\frac{\tilde{\lambda}_{s} \tilde{\rho}_{s} \tilde{c}_{s} \tilde{T}_{0}^{2} \tilde{A}}{\tilde{\dot{q}}^{2}}, \quad \hat{A}=A Q, \quad \hat{r}=\frac{\tilde{\rho}_{g}^{0}}{\tilde{\rho}_{s}}, \quad \hat{b}=\frac{\tilde{c}_{g}}{\tilde{c}_{s}}, \quad \hat{l}=\frac{\tilde{\lambda}_{g}}{\tilde{\lambda}_{s}},
\end{gathered}
$$

where $\tilde{t}$ and $\tilde{\zeta}$ are the time and space variables, and $\tilde{T}$ is the temperature. The initial temperature is denoted by $\tilde{T}_{0}, \tilde{\dot{q}}$ is the external heat flux, $\tilde{\rho}$ and $\tilde{u}$ denote density and velocity, and $\tilde{\lambda}, \tilde{c}$ and $\tilde{Q}$, assumed constant, stand for thermal conductivity, heat capacity (at constant pressure) and heat release, respectively, where subscripts $s$ and $g$ refer to the solid and gaseous phases, and a subscript or superscript 0 denotes evaluation at initial conditions. The kinetic parameters $\tilde{E}$ and $\tilde{A}$ are the activation energy and pre-exponential rate constant, respectively, and $\tilde{R}^{\circ}$ is the gas constant. The ratios $\hat{r}, \hat{b}$ and $\hat{l}$ represent gas-to-solid ratios of density, heat capacity and thermal conductivity, respectively, where the initial gas-phase density $\tilde{\rho}_{g}^{0}$ is used in the definition of $\hat{r}$, and the solid density is assumed constant. Finally, since the nondimensional coordinate $\zeta$ ranges from 0 at the heated surface of the solid to $+\infty$, the appropriate initial and boundary conditions needed to complete the specification of the problem are given by

$$
\left.\left(1-\alpha_{s}+\hat{l} \alpha_{s}\right) \frac{\partial T}{\partial \zeta}\right|_{\zeta=0}= \begin{cases}0, & t<0 \\ -1, & t \geq 0\end{cases}
$$




$$
\begin{gathered}
T(\zeta \rightarrow \infty, t)=T(\zeta, t=0)=1 \\
u_{g}(\zeta \rightarrow 0, t)=0
\end{gathered}
$$

where the closed-end boundary condition (6) replaces the condition $u_{g}(\zeta \rightarrow \infty, t)=0$ used in our previous study of the open-end problem.

As in TMW, we again we seek perturbation solutions of the above problem, based on the fact that $\hat{r} \ll 1$, in the form of the expansions

$T \sim T^{(0)}+\hat{r} T^{(1)}+\cdots, \quad u_{g} \sim u_{g}^{(0)}+\hat{r} u_{g}^{(1)}+\cdots, \quad \rho_{g} \sim \rho_{g}^{(0)}+\hat{r} \rho_{g}^{(1)}+\cdots, \quad t_{c} \sim t_{c}^{(0)}+\hat{r} t_{c}^{(1)}+\cdots$,

where the last of these corresponds to an expansion for the ignition time $t_{c}$, which is defined more precisely below. As before, the primary goal is to describe the effect of gas flow on the time to ignition. Consequently, the analysis outlined below is developed to the point of calculating expressions for the leading-order ignition time $t_{c}^{(0)}$, which depends on porosity and the thermal conductivity of each phase, and the first-order correction $t_{c}^{(1)}$, which contains the first convective effects of gas flow relative to the solid on the ignition-delay time.

\section{Leading-Order Solutions}

By expanding the above problem with respect to $\hat{r}$ according to the expansions introduced in Eqs. (7), the leading order problem in the inert region (where chemical reaction is negligible) is determined as

$$
\begin{gathered}
\frac{\partial \rho_{g}^{(0)}}{\partial t}+\frac{\partial}{\partial \zeta}\left[\rho_{g}^{(0)} u_{g}^{(0)}\right]=0, \quad \rho_{g}^{(0)}=1 / T^{(0)} \\
\left(1-\alpha_{s}\right) \frac{\partial T^{(0)}}{\partial t}=\left(1-\alpha_{s}+\hat{l} \alpha_{s}\right) \frac{\partial^{2} T^{(0)}}{\partial \zeta^{2}}+\hat{A}\left(1-\alpha_{s}\right) e^{-E / T^{(0)}}
\end{gathered}
$$

subject to the leading-order versions of Eqs. (4) - (6). With new space and time variables and the parameters $\hat{A}^{\prime}, a$ and $b$ defined according to

$$
t^{\prime}=\frac{t}{a b}, \quad \zeta^{\prime}=\frac{\zeta}{b}, \quad \hat{A}^{\prime}=a b \hat{A}, \quad a \equiv 1-\alpha_{s}, \quad b \equiv 1-\alpha_{s}+\hat{l} \alpha_{s}
$$

the solution to Eq. (9) can be expressed as

$$
T^{(0)}\left(\zeta^{\prime}, t^{\prime}\right)=T_{I}^{(0)}=1+2 \sqrt{\frac{t^{\prime}}{\pi}} e^{-\zeta^{\prime 2} / 4 t^{\prime}}-\zeta^{\prime} \operatorname{erfc}\left(\zeta^{\prime} / 2 \sqrt{t^{\prime}}\right)
$$

which is the same pure-conduction result obtained for the open-end problem. As before, this solution, which is monotonic in time and maximal at the heated surface $\zeta^{\prime}=0$, becomes invalid for sufficiently large values of time and temperature such that the neglected reaction term in Eq. (2) becomes significant. Critical values of the temperature $T_{c}$ (assumed to be a property of the 
solid material and hence not expanded in powers of $\hat{r}$ ) and $t_{c}^{(0)}$ are therefore introduced such that this is true, where the latter is defined to be the leading-order ignition time introduced in the last of Eqs. (7). Neither are determined precisely at this point, but based on Eq. (11), a relationship between the two is given by

$$
T_{c}=1+2 \sqrt{\frac{t_{c}^{(0)}}{\pi a b}}
$$

or equivalently,

$$
t_{c}^{(0)}=\frac{1}{4} \pi a b\left(T_{c}-1\right)^{2}
$$

The determination of $t_{c}^{(0)}$, and hence $T_{c}$, follows from considering the transition stage described in LW where chemical effects are first felt. Introducing the leading-order departure from the inert solution $\left(\Phi^{(0)}=T^{(0)}-T_{I}^{(0)}\right)$, the energy equation and associated boundary conditions in the transition zone become

$$
\begin{gathered}
\frac{\partial \Phi^{(0)}}{\partial t^{\prime}}=\frac{\partial^{2} \Phi^{(0)}}{\partial \zeta^{2}}+\bar{A}^{\prime} e^{(1 / \epsilon)\left\{\Phi^{(0)}+\left(T_{I}^{(0)}-T_{c}\right)+O\left[\Phi^{(0) 2}, \Phi^{(0)}\left(T_{I}^{(0)}-T_{c}\right),\left(T_{I}^{(0)}-T_{c}\right)^{2}\right]\right\}} \\
\left.\left(\partial \Phi^{(0)} / \partial \zeta^{\prime}\right)\right|_{\left(\zeta^{\prime}=0, t^{\prime}\right)}=\Phi^{(0)}\left(\zeta \rightarrow \infty, t^{\prime}\right)=\Phi^{(0)}\left(\zeta^{\prime}, t^{\prime}=0\right)=0
\end{gathered}
$$

where the small inverse activation-energy parameter $\epsilon=T_{c}^{2} / E \ll \hat{r} \ll 1$ and the scaled rate coefficient $\bar{A}^{\prime}$ are defined as $\bar{A}^{\prime}=a b \bar{A}=a b \hat{A} e^{-E / T_{c}}$. Equations (14) - (15) may then be solved in the limit $\epsilon \rightarrow 0$ according to asymptotic matching principles. In particular, stretched time and space variables are introduced, thereby defining reactive-diffusive (inner) and transient-diffusive (outer) zones such that the solution in the latter is matched to both the inner solution and the inert solution (11), which is still valid in the far outer spatial region. The complete analysis for the nonporous problem was given in LW and reformulated and extended to the porous problem in TMW. This analysis ultimately yields a transition-stage problem whose solution becomes infinite at a finite value of a scaled time, which in turn determines an implicit relation for $t_{c}^{(0)}$ given by

$$
\hat{A}=\frac{\sqrt{E} e^{c_{0}}}{\left(\pi t_{c}^{(0)} / a b\right)^{1 / 4}\left[1+2\left(t_{c}^{(0)} / \pi a b\right)^{1 / 2}\right]} e^{E /\left[1+2\left(t_{c}^{(0)} / \pi a b\right)^{1 / 2}\right]}
$$

Here, $c_{0} \doteq-0.431$ is the finite value of scaled time that leads to blow-up of the transition-stage solution described above. It can be seen that this result is identical to Eq. (29) of LW in the nonporous limit $a b=\left(1-\alpha_{s}\right)\left(1-\alpha_{s}+\hat{l}_{\alpha_{s}}\right)=1$. Hence, as noted in TMW, the relationship between the leading-order critical time for the porous problem and the corresponding critical time in the nonporous limit may be expressed as

$$
t_{c}^{(0)}{ }^{(\text {porous })} \longleftrightarrow\left(1-\alpha_{s}\right)\left(1-\alpha_{s}+\hat{l} \alpha_{s}\right) t_{c}^{(\text {nonporous })} .
$$

When the gas-to-liquid ratio of thermal conductivities obeys the inequality $\hat{l}<\left(2-\alpha_{s}\right) /\left(1-\alpha_{s}\right)$, which is quite realistic since $\left(2-\alpha_{s}\right) /\left(1-\alpha_{s}\right)>2$, it is thus found that, to a first approximation, the 
time to ignition is lessened by the effect of porosity. This is primarily due to the fact that there is simply less solid per unit volume to heat, although the relative rates of thermal conduction clearly enter at this order of approximation as well. To see the effect of gas flow into the solid, however, it is necessary to calculate the next-order correction to the critical time $t_{c}^{(1)}$. It is anticipated, based on the introductory discussion given above, that this coefficient will provide a negative correction to the ignition time for the closed-end problem.

\section{Solution for the Leading-Order Gas Velocity $u_{\mathrm{g}}^{(0)}$}

The inert solution for the leading-order gas velocity follows from Eqs. (8) with the boundary condition given by Eq. (6). Hence, in terms of the primed coordinates and parameters introduced in Eq. (10), the problem for the leading-order gas velocity $u_{g}^{(0)}$ is given by

$$
\begin{gathered}
\frac{1}{a} \frac{\partial \rho_{g}^{(0)}}{\partial t^{\prime}}+\frac{\partial}{\partial \zeta^{\prime}}\left[\rho_{g}^{(0)} u_{g}^{(0)}\right]=0 \\
u_{g}^{(0)}\left(\zeta^{\prime} \rightarrow 0, t^{\prime}\right)=0
\end{gathered}
$$

where $\rho_{g}^{(0)}=1 / T^{(0)}$ is known from Eq. (11), and we have now dropped the subscript "I" denoting the solution in the inert regime. Performing an integration with respect to $\zeta^{\prime}$ thus yields

$$
\begin{aligned}
u_{g}^{(0)}\left(\zeta^{\prime}, t^{\prime}\right) & =-\frac{1}{a \rho_{g}^{(0)}\left(\zeta^{\prime}, t^{\prime}\right)} \int_{0}^{\zeta^{\prime}} \frac{\partial \rho_{g}^{(0)}\left(\zeta^{*}, t^{\prime}\right)}{\partial t^{\prime}} d \zeta^{*} \\
& =a^{-1} T^{(0)}\left(\zeta^{\prime}, t^{\prime}\right) \int_{0}^{\zeta^{\prime}}\left[T^{(0)}\left(\zeta^{*}, t^{\prime}\right)\right]^{-2} \frac{\partial T^{(0)}\left(\zeta^{*}, t^{\prime}\right)}{\partial t^{\prime}} d \zeta^{*}
\end{aligned}
$$

In view of the expression for the inert temperature solution $T^{(0)}$ given by Eq. (11), the gas velocity therefore can be expressed as a function of the scaled temporal and spatial variables in the inert region as

$$
u_{g}^{(0)}=\frac{1}{a \sqrt{\pi t^{\prime}}}\left[1+2 \sqrt{\frac{t^{\prime}}{\pi}} e^{-\zeta^{\prime 2} / 4 t^{\prime}}-\zeta^{\prime} \operatorname{erfc}\left(\frac{\zeta^{\prime}}{2 \sqrt{t^{\prime}}}\right)\right] \int_{0}^{\zeta^{\prime}} \frac{e^{-\zeta^{-2} / 4 t^{\prime}} d \zeta^{*}}{\left[1+2 \sqrt{\frac{t^{\prime}}{\pi}} e^{-\zeta^{* 2} / 4 t^{\prime}}-\zeta^{*} \operatorname{erfc}\left(\frac{\zeta^{*}}{2 \sqrt{t^{\prime}}}\right)\right]^{2}},
$$

which differs from the corresponding result for the open-end problem only in the limits of integration on the integral in Eq. (21).

The profiles of the inert gas velocity for various times are shown in Figures 2a,b for two different values of the porosity $\alpha_{s}$ in terms of the original nondimensional physical variables $(\zeta, t)$. The profiles demonstrate that the gas velocity is positive (i.e., directed into the porous solid). At any fixed time, the gas velocity achieves it's largest magnitude far in the sample, while for any fixed spatial location and time the magnitude of $u_{g}^{(0)}$ is larger for higher porosities. The latter 
occurs because for higher porosities, the solid is heated more rapidly, resulting in a greater degree of gas-phase thermal expansion at any given instant of time.

It is also observed that, contrary to the result for the open-end problem, $u_{g}^{(0)}$ initially achieves an instantaneous nonzero value, uniform throughout the sample, which then relaxes in time. Indeed, for small times $\left(t^{\prime} \ll 1\right)$, the expression $(21)$ for $u_{g}^{(0)}$ may be simplified, giving rise to the approximate result

$$
u_{g}^{(0)} \sim \frac{1}{a \sqrt{\pi t^{\prime}}} \int_{0}^{\zeta^{\prime}} e^{-\zeta^{* 2} / 4 t^{\prime}} d \zeta^{*}=\frac{1}{a} \operatorname{erf}\left[\frac{\zeta^{\prime}}{2 \sqrt{t^{\prime}}}\right], \quad t^{\prime} \ll 1
$$

Clearly, for all $\zeta^{\prime} \gg \mathrm{O}\left(\sqrt{t^{\prime}}\right)$, we have $u_{g}^{(0)} \sim a^{-1}$. This is the instantaneous value that the gas velocity is found to reach throughout the sample as soon as heating commences, although since the gas is initially at rest, there must, in fact, be a period of time during which $u_{g}^{(0)}$ increases from zero to $a^{-1}$. The absence of such a period stems from the assumption of constant pressure, which effectively allows a gasdynamic signal to propagate infinitely fast. In reality, a pressure wave would initially propagate through the gas phase at the local speed of sound such that ahead of the wave the gas velocity would still be zero, and behind it the above value would be approached as the wave passed. Reasonable parameter restrictions that justify the neglect of these and certain other effects, such as Darcy-type flow resistance, are given in the Appendix.

\section{First-Order Corrections $\mathbf{T}^{(\mathbf{1})}$}

In order to proceed further in the analysis, it is necessary to calculate the next-order correction to the inert temperature, namely the coefficient $T^{(1)}$ in the first of Eqs. (7). For this purpose, from Eqs. (2), (4) and (5), the problem for $T^{(1)}$ is obtained as

$$
\begin{aligned}
& \frac{\partial}{\partial t}\left[\left(1-\alpha_{s}\right) T^{(1)}+\hat{b} \alpha_{s} u_{g}^{(0)}\right]+\hat{b} \frac{\partial}{\partial \zeta}\left[\alpha_{s} u_{g}^{(0)}\right]= \\
& \quad \frac{\partial}{\partial \zeta}\left[\left(1-\alpha_{s}+\hat{l} \alpha_{s}\right) \frac{\partial T^{(1)}}{\partial \zeta}\right]+\hat{A} E\left(1-\alpha_{s}\right) \frac{T^{(1)}}{T^{(0)}{ }^{2}} e^{-E / T^{(0)}} \\
& \left.\frac{\partial T^{(1)}}{\partial \zeta}\right|_{\zeta=0}=0, \quad T^{(1)}(\zeta \rightarrow \infty, t)=T^{(1)}(\zeta, t=0)=0
\end{aligned}
$$

where the reaction term in Eq. (23) is obtained from the expansion of the Arrhenius factor $e^{-E / T}$ with respect to $\hat{r}$ when the first of the expansions (7) is substituted for $T$. We observe that at this (and higher) orders the boundary conditions (24) are homogeneous.

As in the leading-order analysis, the inert and transition-zone stages are considered, where the latter is characterized by the same reactive-diffusive (inner), transient-diffusive (outer) and inert 
(far outer) spatial zones as before. In the inert regime, Eq. (23) reduces to a nonhomogeneous heat-conduction equation given by

$$
\frac{\partial T^{(1)}}{\partial t^{\prime}}-\frac{\partial^{2} T^{(1)}}{\partial \zeta^{2}}=-\hat{b} \alpha_{s}\left[\frac{1}{a} \frac{\partial u_{g}^{(0)}}{\partial t^{\prime}}+\frac{\partial u_{g}^{(0)}}{\partial \zeta^{\prime}}\right]
$$

subject to the boundary conditions (24). The nonhomogeneous right-hand side of Eq. (25), which we denote by $g\left(\zeta^{\prime}, t^{\prime}\right)$, involves first derivatives of $u_{g}^{(0)}$ that are calculated from the result given by Eq. (21). Introducing the appropriate Green's function for the above problem, namely

$$
G\left(\zeta^{\prime}, t^{\prime} \mid x, \sigma\right)=\frac{1}{\sqrt{4 \pi\left(t^{\prime}-\sigma\right)}}\left[e^{-\left(\zeta^{\prime}-x\right)^{2} / 4\left(t^{\prime}-\sigma\right)}+e^{-\left(\zeta^{\prime}+x\right)^{2} / 4\left(t^{\prime}-\sigma\right)}\right]
$$

the solution of the first-order inert problem can be expressed as

$$
T^{(1)}\left(\zeta^{\prime}, t^{\prime}\right)=\int_{0}^{t^{\prime}} \int_{0}^{\infty} G\left(\zeta^{\prime}, t^{\prime} \mid x, \sigma\right) g(x, \sigma) d x d \sigma
$$

This integral has been evaluated numerically to obtain the profiles $T^{(1)}$ as a function of the original physical variable $\zeta$ for different porosities at various values of the nondimensional physical time $t$, as shown in Figures $3 \mathrm{a}, \mathrm{b}$. It is readily seen that the correction $T^{(1)}$ to the leading-order inert temperature is positive, which stems from the fact that gas flows into the porous solid, providing a preheating effect. Initially, this correction is more significant near the heated surface, and it decays farther into the sample. As time increases, however, the maximum correction moves deeper into the material, resulting in a thermal wave propagating into the sample. Thus, unlike the diffusive heat-transfer process, the convective aspect of heat transfer resulting from the two-phase flow is not spatially monotonic. Figure 4 demonstrates that $T^{(1)}$ increases with porosity for fixed values of the space and time coordinates.

As with the leading-order solution $T^{(0)}$, the inert solution for $T^{(1)}$ also becomes invalid in the vicinity of the ignition time $t_{c}$ because of the onset of chemical reaction. In particular, the transition-stage analysis at this order determines that the first-order solution becomes infinite at the same finite value of the scaled transition time variable as the leading-order solution (cf. TMW). Consequently, the relationship (16) remains valid, and the correction coefficient $t_{c}^{(1)}$ to the ignition time can be calculated based on the two-term expansion for the inert temperature as shown below.

\section{Calculation of the Correction to the Ignition-Delay Time $t_{c}^{(1)}$}

Since the ignition temperature $T_{c}$ has been regarded as a property of the pure material, it does not depend on $\hat{r}$, and thus the two-term ignition condition at the solid surface is expressed as

$$
T_{c} \approx T^{(0)}\left(0, t_{c}^{\prime}\right)+\hat{r} T^{(1)}\left(0, t_{c}^{\prime}\right)
$$


where, from the last of Eqs. (7) and the first of Eqs. (10), $t_{c}^{\prime} \sim t_{c}^{\prime(0)}+\hat{r}_{c}^{\prime(1)}+\cdots$. Since $t_{c}^{(0)}$ and $T_{c}$ are determined from Eqs. (12) and (16), Eq. (28) with the above expansion for $t_{c}^{\prime}$ constitutes an implicit relationship for $t_{c}^{(1)}$. Defining $h_{0}\left(t_{c}^{\prime}\right)=T^{(0)}\left(0, t_{c}^{\prime}\right)=1+2 \sqrt{t_{c}^{\prime} / \pi}$, and $h_{1}\left(t_{c}^{\prime}\right)=T^{(1)}\left(0, t_{c}^{\prime}\right)$, where the latter is obtained from Eq. (27), and substituting the expansion for $t_{c}^{\prime}$ into Eq. (28), we obtain

$$
\begin{aligned}
T_{c} & \approx h_{0}\left(t_{c}^{\prime(0)}+\hat{r} t_{c}^{\prime(1)}+\cdots\right)+\hat{r} h_{1}\left(t_{c}^{\prime(0)}+\hat{r} t_{c}^{(1)}+\cdots\right) \\
& \sim h_{0}\left(t_{c}^{\prime(0)}\right)+\hat{r}\left[h_{1}\left(t_{c}^{\prime(0)}\right)+t_{c}^{\prime(1)} d h_{0} /\left.d t_{c}^{\prime}\right|_{t_{c}^{\prime}=t_{c}^{\prime(0)}}\right] .
\end{aligned}
$$

Consequently, the leading-order result $T_{c}=h_{0}\left(t_{c}^{\prime(0)}\right)$ is recovered, while at the next order, an explicit expression for $t_{c}^{\prime(1)}$ is obtained as

$$
t_{c}^{\prime(1)}=-\frac{h_{1}\left(t_{c}^{\prime(0)}\right)}{d h_{0} /\left.d t_{c}^{\prime}\right|_{t_{c}^{\prime}=t_{c}^{\prime}}{ }^{(0)}} .
$$

From the above definitions for $h_{0}$ and $h_{1}$, the final result becomes

$$
t_{c}^{\prime(1)}=\sqrt{t_{c}^{\prime(0)}} \int_{0}^{t_{c}^{\prime(0)}} \int_{0}^{\infty} \frac{e^{-x^{2} / 4\left(t_{c}^{\prime(0)}-\sigma\right)}}{\sqrt{t_{c}^{\prime(0)}-\sigma}} g(x, \sigma) d x d \sigma .
$$

The above multiple integral was evaluated numerically to determine the correction coefficient $t_{c}^{(1)}$ as a function of porosity. In particular, Eq.(16) was evaluated for each value of porosity to determine the leading-order ignition time $t_{c}^{(0)}$, using the physical parameters for HMX from Li et al. (1990) and Bradley (1970). The corresponding value for $t_{c}^{(0)}$ was then substituted into Eq. (31) to provide the final result, which is shown in Figure 5. It is readily seen that the correction to the ignition time is negative. This result is consistent with the physical expectation that a flow of gas through the solid, in the same direction as the heat flux, speeds up the ignition process by providing a convective transport mechanism that supplements the conductive transfer of heat from the surface into the porous solid. As the porosity increases, the magnitude of $t_{c}^{(1)}$ increases as well. Thus, the ignition time is shortened by an increase in porosity through two effects. First, at leading order, there is less reactant, so for the same rate of external energy applied to the surface, a more porous solid will achieve the ignition temperature before a less porous one, as quantitatively expressed by Eq. (17). At the next order, the positive flow of gas into the solid provides a convective preheating mechanism that shortens the ignition time even further. This last result is opposite to that obtained in TMW for the open-end problem, in which case the flow of gas out of the system and in the opposite direction of the applied energy flux had the effect of removing energy and thereby delaying the ignition time relative to the leading-order result

\section{Conclusion}

The present study represents a continuation of the ignition analysis of a porous energetic material initiated in Part I (Telengator, Margolis, Williams, 1998). In the present analysis, the 
heated surface of the material was assumed to be closed, resulting in a thermal-expansion-induced gas flow into the porous solid that provides a preheating effect and shortens the time to ignition relative to the leading-order result, which depends only on porosity and thermal conductivity. This result is opposite to that which was obtained for the open-end problem, in which case the flow of gas is directed out of the solid, thereby removing energy from the system and thus increasing the ignition-delay time relative to the leading-order expression. Consequently, the correction to the inert temperature is positive near the closed heated surface, as opposed to the negative temperature correction obtained for the problem in which the heated surface was open. Another consequence of the closed-end condition is that the nondimensional gas velocity reaches a finite positive value $\left(1-\alpha_{s}\right)^{-1}$ throughout the sample within a very short time, since thermally expanded gas in the vicinity of the surface cannot escape and must therefore flow through the porous solid. In the constant-pressure limit, this velocity is felt instantaneously far into the sample, although the rate at which the velocity signal can propagate is physically limited either by the speed of sound or the viscous resistance experienced by the gas. In both types of problems, the leading-order effect of porosity is to shorten the ignition-delay time, thus raising safety concerns when an aged or degraded energetic material develops significant porosity. Since the closed-end condition further shortens the ignition delay, unlike the open-end condition, the closed end may be considered to worsen the hazard for a given value of the heat-flux stimulus.

\section{Appendix. Acoustic and Flow-Resistance Effects}

Reasonable restrictions on the present problem that justify the neglect of the acoustic and flow-resistance effects mentioned in the text may be deduced as follows. A characteristic time scale appropriate for describing the initial period of sound propagation is $\tilde{\lambda}_{s} \tilde{T}_{0} /\left(\tilde{a}_{0} \tilde{\tilde{q}}\right)$, where $\tilde{a}_{0}$ is the speed of sound in the ambient gas, since according to the length scaling in Eq. (3), the thickness of the heated layer is of order $\tilde{\lambda}_{s} \tilde{T}_{0} / \tilde{\dot{q}}$. This time must be short compared with the ignition time, which is of order $\tilde{\lambda}_{s} \tilde{\rho}_{s} \tilde{c}_{s} \tilde{T}_{0}^{2}\left(1-\alpha_{s}\right) / \tilde{\dot{q}}^{2}$, where the porosity factor $\left(1-\alpha_{s}\right)$, suggested by the first of Eqs. (10), arises from the fact that a given external heat flux will heat a porous solid faster than its nonporous counterpart. Consequently, these estimates impose the restriction $\tilde{a}_{0} \tilde{\rho}_{s} \tilde{c}_{s} \tilde{T}_{0} \gg \tilde{\dot{q}} /\left(1-\alpha_{s}\right)$. From a different viewpoint, the velocity scaling in Eq. (3) and the first of Eqs. (10) indicate that the Mach number of the gas is of order $\tilde{\dot{q}} /\left[\tilde{\rho}_{s} \tilde{c}_{s} \tilde{T}_{0} \tilde{a}_{0}\left(1-\alpha_{s}\right)\right]$, and since this Mach number must be small for the constant-pressure approximation to apply, the same condition $\tilde{a}_{0} \tilde{\rho}_{s} \tilde{c}_{s} \tilde{T}_{0} \gg \tilde{\dot{q}} /\left(1-\alpha_{s}\right)$ is obtained. Typical values of $\tilde{a}_{0}$ are sufficiently large that, unless the external heat flux $\tilde{\dot{q}}$ is extremely large or the porosity $\alpha_{s}$ is very near unity, effects arising from the propagation of the pressure wave may be neglected.

Finally, a separate requirement for the neglect of pressure gradients in the ignition layer of interest, applicable to the previous problem (TMW) as well as to the present one, can be derived 
from a Darcy-law restriction on the maximum gas-flow resistance of the porous medium. Thus, in a porous medium, the pressure drop $\Delta \tilde{p}_{g}$ across a distance $\Delta \tilde{x}$ may be empirically expressed according to Darcy's law as $\alpha_{s} \tilde{\mu}_{g} \tilde{u}_{g} \Delta \tilde{x} / \tilde{\kappa}\left(\alpha_{s}\right)$, where $\tilde{\mu}_{g}$ is the viscosity of the gas and $\tilde{\kappa}\left(\alpha_{s}\right)$ is the porosity-dependent permeability of the porous material (typically, the product $\alpha_{s}^{-1} \tilde{\kappa}\left(\alpha_{s}\right)$ is a decreasing function of $\alpha_{s}$, so that a larger pressure drop is required to maintain a given gas velocity as the porosity decreases; $c f$. Probstein, 1989). Taking the distance $\Delta \tilde{x}$ to be the thickness of the heated layer $\tilde{\lambda}_{s} \tilde{T}_{0} / \tilde{\dot{q}}$ and the velocity $\tilde{u}_{g}$ to be the characteristic velocity $\tilde{\dot{q}} /\left[\tilde{\rho}_{s} \tilde{c}_{s} \tilde{T}_{0}\left(1-\alpha_{s}\right)\right]$, the assumption that the relative pressure perturbation $\Delta \tilde{p}_{g} / \tilde{p}_{g}^{0}$ be small leads to the condition $\tilde{\mu}_{g} \tilde{\lambda}_{s} \tilde{W}_{g} /\left[\left(1-\alpha_{s}\right) \alpha_{s}^{-1} \tilde{\kappa}\left(\alpha_{s}\right) \tilde{c}_{s} \tilde{\rho}_{s} \tilde{\rho}_{g}^{0} \tilde{R}^{\circ} \tilde{T}_{0}\right] \ll 1$, where, having used the gas-phase equation of state, $\tilde{W}_{g}$ is the molecular weight of the gas. Since the kinematic viscosity $\tilde{\mu}_{g} / \tilde{\rho}_{g}^{0}$ is typically small, this condition is violated only when the permeability of the material is sufficiently small as well.

Since $\alpha_{s}^{-1} \tilde{\kappa}\left(\alpha_{s}\right)=\tilde{r}_{p}^{2}$, where $\tilde{r}_{p}$ is a suitable representative transverse pore dimension, the preceding inequality essentially requires that the product of a gas-phase momentum diffusivity $\tilde{\mu}_{g} / \tilde{\rho}_{g}^{0}$ and a solid-phase thermal diffusivity $\tilde{\lambda}_{s} /\left(\tilde{c}_{s} \tilde{\rho}_{s}\right)$ be small compared with the product of $\tilde{r}_{p}^{2}$ and the square of a representative gas-phase molecular velocity, $\left(\tilde{R}^{\circ} \tilde{T}_{0} / \tilde{W}_{g}\right)^{1 / 2}$. This result is effectively equivalent to equating the pressure-gradient and viscous terms in momentum conservation and requiring that the resulting pressure change be small compared to the absolute pressure $\tilde{p}_{g}^{0}$. Additional information, however, is obtained by comparing the transient and inertial terms in that equation with the viscous term, where the latter is of order $\tilde{\mu}_{g} \tilde{u}_{g} / \tilde{r}_{p}^{2}$. From the estimates given above, the former two terms are both of order $\tilde{\rho}_{g}^{0} \tilde{u}_{g}^{2} / \Delta \tilde{x}=\tilde{\rho}_{g}^{0} \tilde{u}_{g} \tilde{\dot{q}}^{2} /\left[\tilde{\lambda}_{s} \tilde{\rho}_{s} \tilde{c}_{s} \tilde{T}_{0}^{2}\left(1-\alpha_{s}\right)\right]$, where the latter equality was obtained by substituting the characteristic ignition time $\tilde{t}_{I}=\tilde{\lambda}_{s} \tilde{\rho}_{s} \tilde{c}_{s} \tilde{T}_{0}^{2}\left(1-\alpha_{s}\right) / \tilde{\dot{q}}^{2}$ for $\left(\tilde{u}_{g} / \Delta \tilde{x}\right)^{-1}$. For the viscous term not to be large compared with the transient and inertial terms, it is therefore necessary to impose the condition that $\tilde{\mu}_{g} \tilde{\lambda}_{s} \tilde{\rho}_{s} \tilde{c}_{s} \tilde{T}_{0}^{2}\left(1-\alpha_{s}\right) /\left[\alpha_{s}^{-1} \tilde{\kappa}\left(\alpha_{s}\right) \tilde{\rho}_{g}^{0} \tilde{\dot{q}}^{2}\right] \lesssim 1$, or $\left(\tilde{\mu}_{g} / \tilde{\rho}_{g}\right)\left[\tilde{\lambda}_{s} /\left(\tilde{\rho}_{s} \tilde{c}_{s}\right)\right] \lesssim \alpha_{s}^{-1} \tilde{\kappa}\left(\alpha_{s}\right) \tilde{\dot{q}}^{2} /\left[\tilde{\rho}_{s}^{2} \tilde{c}_{s}^{2} \tilde{T}_{0}^{2}\left(1-\alpha_{s}\right)\right] \sim \tilde{r}_{p}^{2} \tilde{u}_{g}^{2}$, where the last relation is-deduced from the velocity scaling based on Eqs. (3) and (10). This inequality is more restrictive than the preceding one by a factor of the Mach number squared, in that it requires the same product of gas-phase and solid-phase diffusivities not to exceed the product of $\tilde{r}_{p}^{2}$ with the square of the gas velocity $\tilde{u}_{g}$. Combined with the acoustic restriction indicated above, this condition prevents the incident energy flux from being either too small or too large. In particular,

$$
\frac{\tilde{\mu}_{g}}{\tilde{\rho}_{g}^{0}} \cdot \frac{\tilde{\lambda}_{s}}{\tilde{\rho}_{s} \tilde{c}_{s}} \lesssim \frac{\tilde{r}_{p}^{2}}{1-\alpha_{s}}\left(\frac{\tilde{\dot{q}}}{\tilde{\rho}_{s} \tilde{c}_{s} \tilde{T}_{0}}\right)^{2} \ll \tilde{r}_{p}^{2}\left(1-\alpha_{s}\right) \tilde{a}_{0}^{2},
$$

which, if a Darcy time $\tilde{t}_{D}$ is defined as $\tilde{t}_{D}=\tilde{r}_{p}^{2} \tilde{\rho}_{g}^{0} / \tilde{\mu}_{g}$, requires the characteristic ignition time $\tilde{t}_{I}$ to satisfy

$$
\tilde{t}_{D}^{-1} \lesssim \tilde{t}_{I}^{-1} \ll\left(\tilde{t}_{I} / \tilde{t}_{A}\right) \tilde{t}_{A}^{-1}
$$

where $\tilde{t}_{A}=\tilde{\lambda}_{s} \tilde{T}_{0} /\left(\tilde{a}_{0} \tilde{\dot{q}}\right)$ is the acoustic time scale introduced above that characterizes the initial period of sound propagation. The ignition time is thus restricted to be less than the Darcy time 
but much greater than the acoustic time. With typical real values of the various parameters, these restrictions are satisfied and enable $\tilde{\dot{q}}$ to range over at least two orders of magnitude.

\section{References}

1. Bradley, H. H., 1970, Theory of Ignition of a Reactive Solid by Constant Energy Flux, Combust. Sci. Tech. 2, pp. 11-20.

2. Kapila, A. K., 1981, Evolution of Deflagration in a Cold Combustible Subjected to a Uniform Energy Flux, Int. J. Eng. Sci. 19, pp. 495-509.

3. Kassoy, D. R., and Poland, J., 1980, The Thermal Explosion Confined by a Constant Temperature Boundary: I-The Induction Period Solution, SIAM J. Appl.Math. 39, pp. 412-430.

4. Leiper, G. A., and Roach, G. F., 1993, On the Theory of Ignition of a Reactive Solid by a Non-Constant Energy Flux, Proceedings Tenth International Detonation Symposium, Boston, pp. 856-861.

5. Kassoy, D. R., and Poland, J., 1981, The Thermal Explosion Confined by a Constant Temperature Boundary: I-The Extremely Rapid Transient, SIAM J. Appl.Math. 41, pp. 231-246.

6. Li, S. C., Williams, F. A., and Margolis, S. B., 1990, Effects of Two-Phase Flow in a Model for Nitramine Deflagration, Combust. Flame 80, pp. 329-349.

7. Liñán, A., and Williams, F. A., 1971, Theory of Ignition of a Reactive Solid by a Constant Energy Flux, Combust. Sci. Tech. 3, pp. 91-98.

8. Margolis, S. B., and Williams, F. A., 1995a, Effects of Two-Phase Flow on the Deflagration of Porous Energetic Materials, J. Propulsion Power 11, pp. 759-768.

9. Margolis, S. B., and Williams, F. A., 1995b, Influence of Porosity and Two-Phase Flow on Diffusional/Thermal Instability of a Deflagrating Energetic Material, Combust. Sci. Tech. 106, pp. 41-68.

10. Margolis, S. B., and Williams, F. A., 1996, Effect of Gas-Phase Thermal Expansion on Stability of Deflagrations in Porous Energetic Materials, Int. J. Multiphase Flow 22, pp. 69-91.

11. Probstein, R. F., 1989, Physicochemical Hydrodynamics-An Introduction, Butterworth Publishers, Boston, pp. 98-100.

12. Telengator, A.M., Margolis, S.B., and Williams, F.A., 1998, Analysis of Ignition of a Porous Energetic Material, Combust. Theory Modelling, to appear.

13. Williams, F. A., 1985, Combustion Theory, Second Edition, Benjamin/Cummings, Menlo Park.

14. Zel'dovich, Ya. B., Barenblatt, G. I., Librovich, V. B., and Makhviladze, G. M., 1985, The Mathematical Theory of Combustion and Explosions, Consultants Bureau, New York. 


\section{Figure Captions}

Figure 1. Physical description of the problem. A constant heat flux is applied to one surface of a semi-infinite porous energetic solid, leading to thermal runaway (ignition) in finite time. The heated end is closed to gas flow.

Figure 2. Leading-order gas velocity profile $u_{g}^{(0)}$ as a function of the spatial coordinate $\zeta$, for several values of time; (a) $\alpha_{s}=0.2, \hat{l}=0.9$; (b) $\alpha_{s}=0.45, \hat{l}=0.9$.

Figure 3. Correction coefficient $T^{(1)}$ in the inert region as a function of $\zeta$ for several values of time; (a) $\alpha_{s}=0.1, \hat{b}=1.0$; (b) $\alpha_{s}=0.4, \hat{b}=1.0$.

Figure 4. Correction coefficient $T^{(1)}$ in the inert region as a function of porosity $\alpha_{s}$ for two different values of space and time $(\hat{b}=1.0)$.

Figure 5. Correction coefficient $t_{c}^{(1)}$ as a function of the porosity $\alpha_{s}$, for two values of the gas-tosolid thermal conductivity ratio $\hat{l}\left(\hat{b}=1.0, Q=8.0, E=77.5\right.$ and $\hat{A}=4.72 \times 10^{16}$, where the latter two quantities were calculated from Eq. (1) using the dimensional values $\tilde{E}=46,200 \mathrm{cal} / \mathrm{mole}, \tilde{T}_{0}=298^{\circ} \mathrm{K}, \tilde{A}=5 \times 10^{15} / \mathrm{sec}, \tilde{\lambda}_{s}=5 \times 10^{-4} \mathrm{cal} / \mathrm{cm} / \mathrm{sec} /{ }^{\circ} \mathrm{K}$, $\tilde{\rho}_{s}=1.9 \mathrm{~g} / \mathrm{cm}^{3}, \tilde{c}_{s}=0.35 \mathrm{cal} / \mathrm{g} /{ }^{\circ} \mathrm{K}$ and $\left.\tilde{\dot{q}}=5.0 \mathrm{cal} / \mathrm{cm}^{2} / \mathrm{sec}\right)$. 


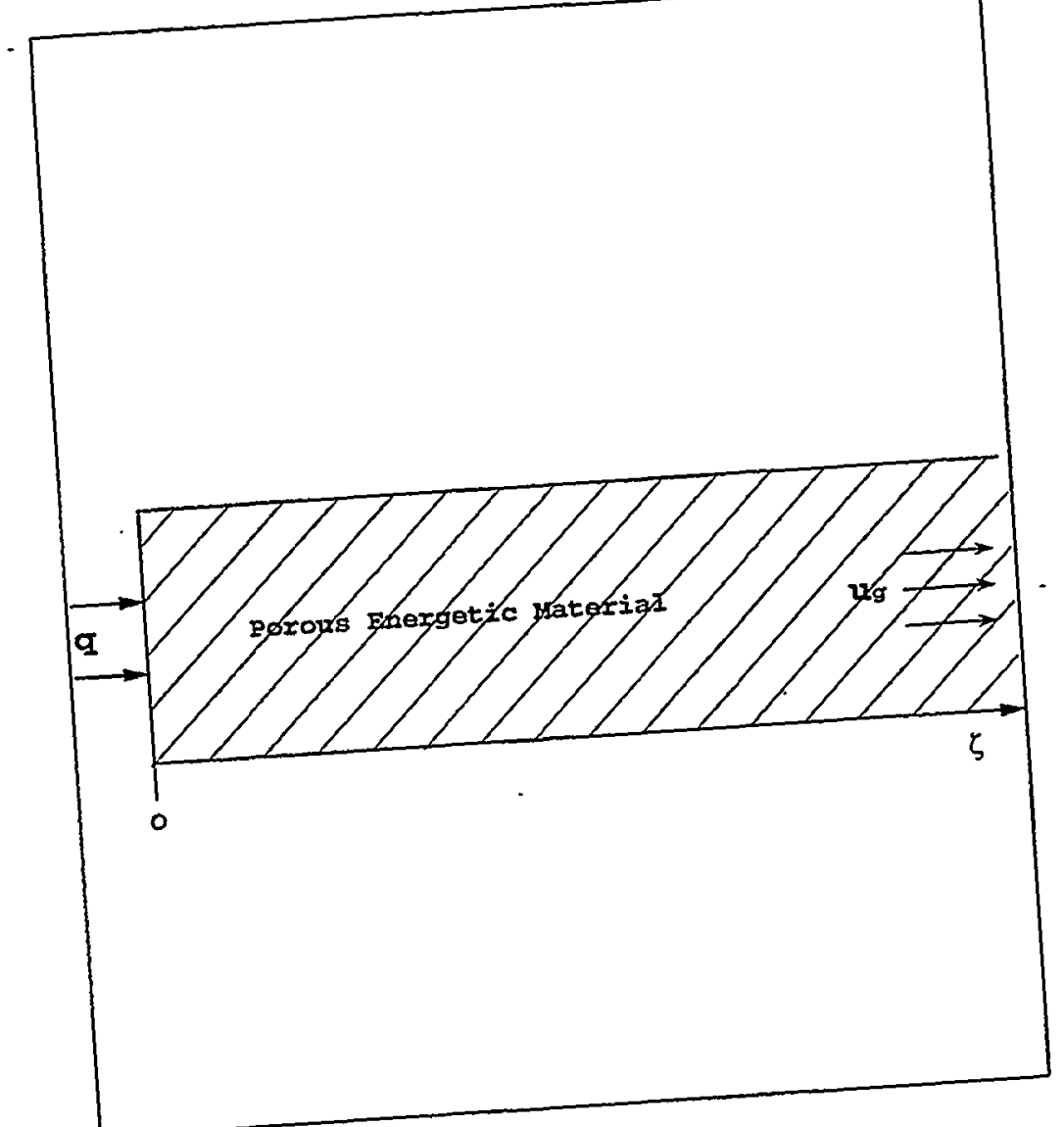

Figure 1. Physical description of the problem. A constant energy flux is applied to one surface of a semi-infinite porous energetic solid, leading to thermal runaway (ignition) in finite time. The heated end is closed to gas flow. 

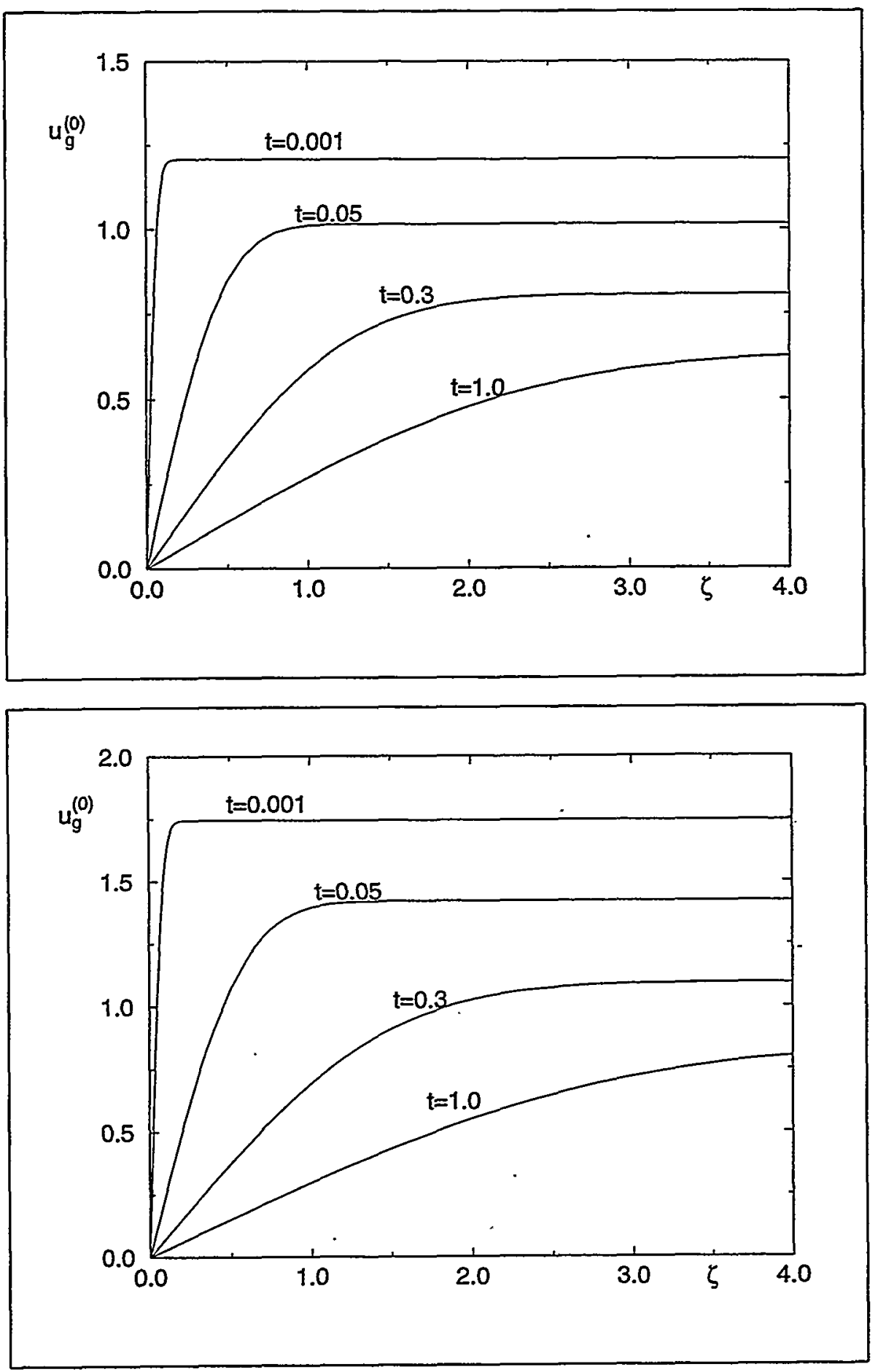

Figure 2. Leading-order gas velocity profile $u_{g}^{(0)}$ as a function of the spatial coordinate $\zeta$, for several values of time; (a, top) $\alpha_{s}=0.2, \hat{l}=0.9 ;\left(b\right.$, bottom) $\alpha_{s}=0.45, \hat{l}=0.9$. 

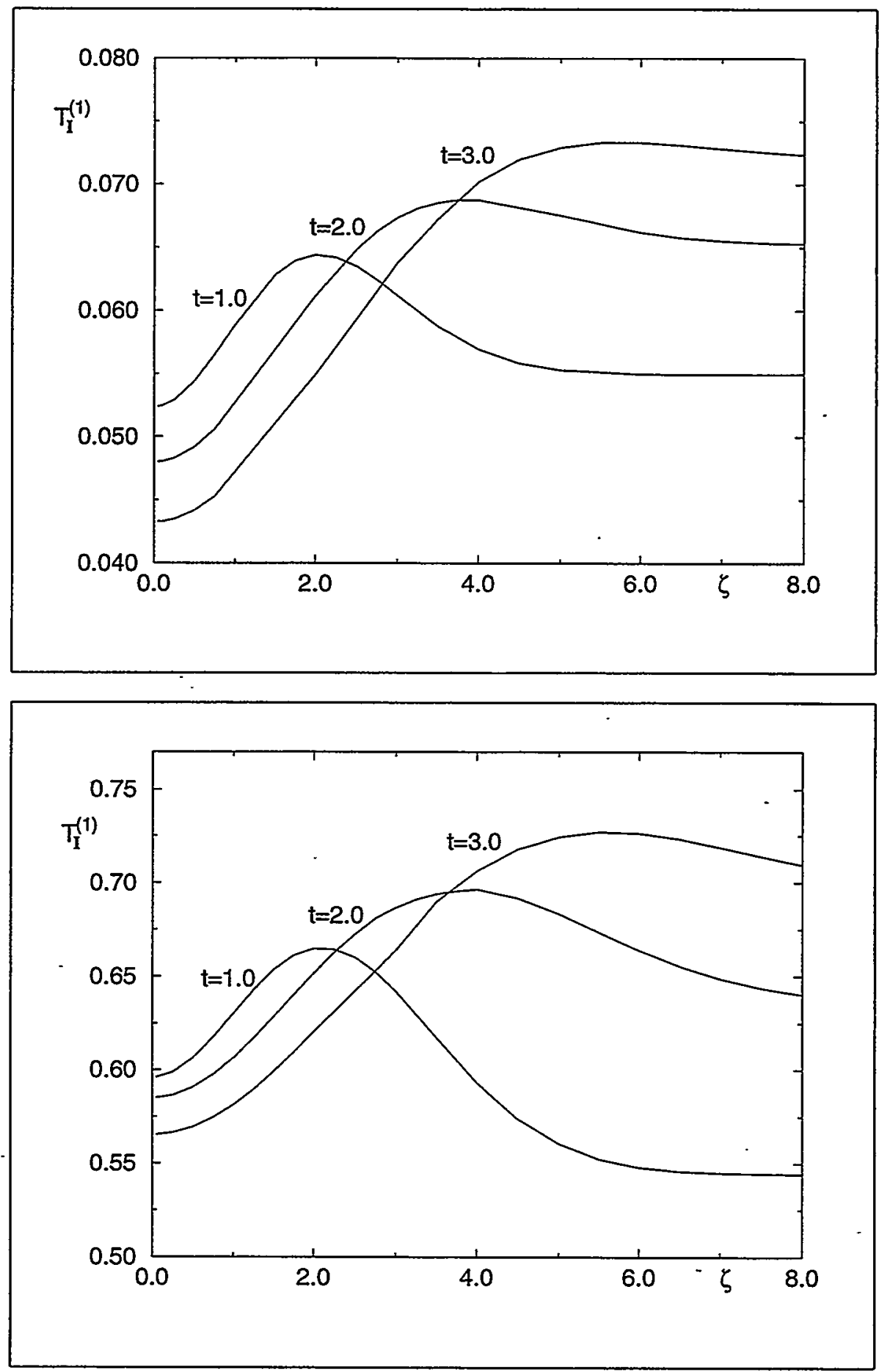

Figure 3. Correction coefficient $T_{I}^{(1)}$ in the inert region as a function of $\zeta$ for several values of time; (a, top) $\alpha_{s}=0.1, \hat{b}=1.0 ;\left(b\right.$, bottom) $\alpha_{s}=0.4, \hat{b}=1.0$. 


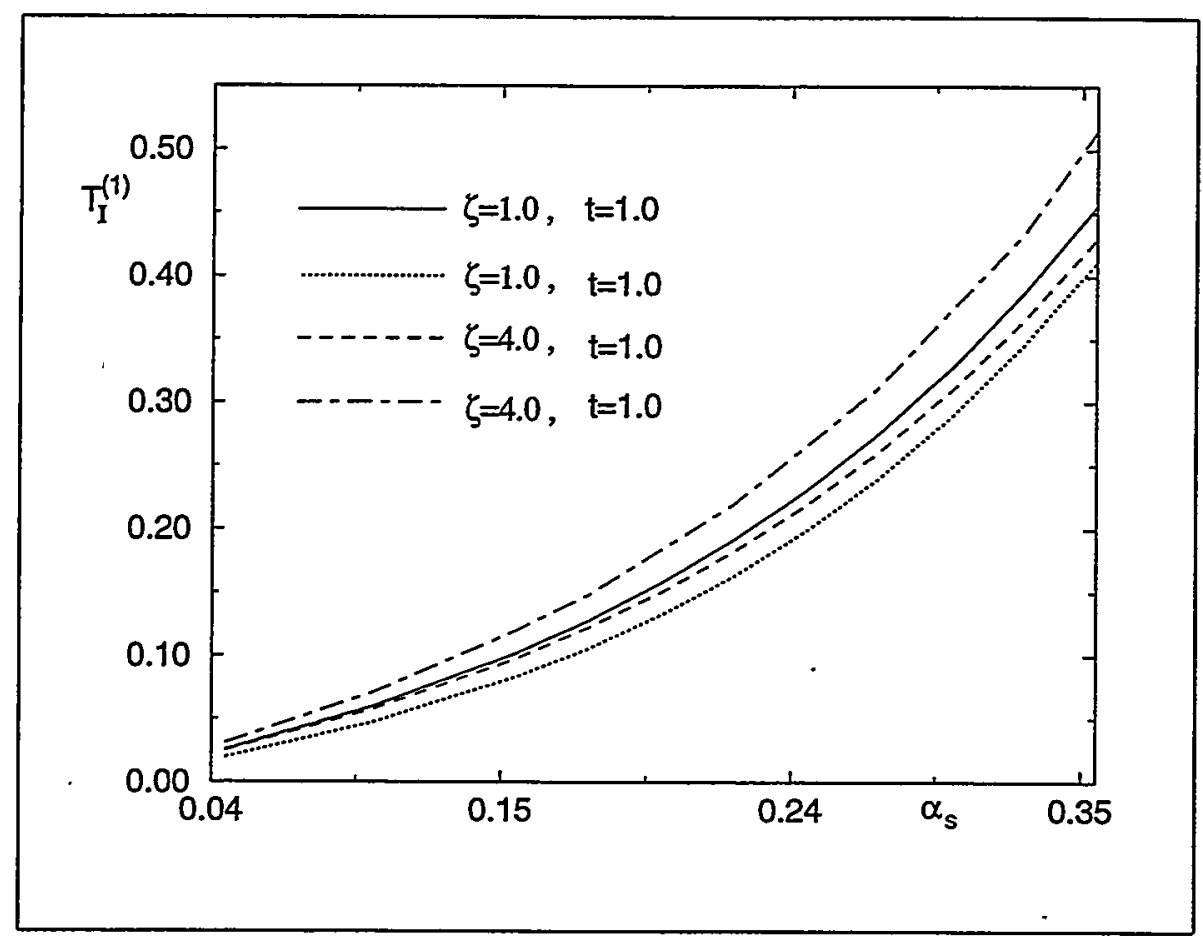

Figure 4. Correction coefficient $T^{(1)}$ in the inert region as a function of porosity $\alpha_{s}$ for two different values of space and time $(\hat{b}=1.0)$. 


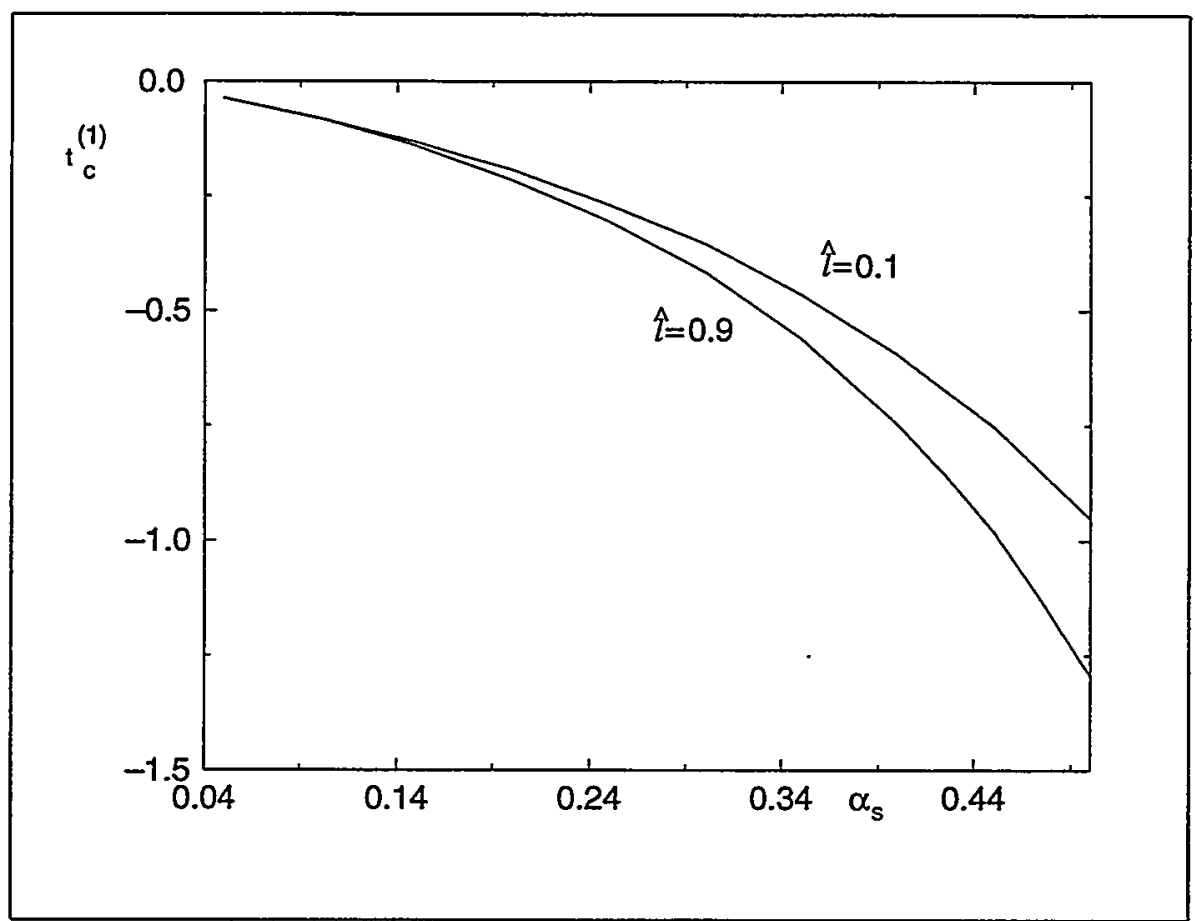

Figure 5. Correction coefficient $t_{c}^{(1)}$ as a function of the porosity $\alpha_{s}$, for two values of the gas-tosolid thermal conductivity ratio $\hat{l}\left(\hat{b}=1.0, Q=8.0, E=77.5\right.$ and $\hat{A}=4.72 \times 10^{16}$, where the latter two quantities were calculated from $E q$. (1) using the dimensional values $\tilde{E}=46,200 \mathrm{cal} / \mathrm{mole}$, $\tilde{T}_{0}=298^{\circ} \mathrm{K}, \tilde{A}=5 \times 10^{15} / \mathrm{sec}, \tilde{\lambda}_{s}=5 \times 10^{-4} \mathrm{cal} / \mathrm{cm} / \mathrm{sec} / \mathrm{K}_{,} \tilde{\rho}_{s}=1.9 \mathrm{~g} / \mathrm{cm}^{3}, . \tilde{c}_{s}=0.35 \mathrm{cal} / \mathrm{g} /{ }^{\circ} \mathrm{K}$ and $\tilde{\dot{q}}=5.0 \mathrm{cal} / \mathrm{cm}^{2} / \mathrm{sec}$ ). 


\section{UNLIMITED RELEASE}

\section{INITIAL DISTRIBUTION}

Dr. John K. Bechtold

Department of Mathematics

New Jersey Institute of Technology

Newark, NJ 07102-1982

Dr. Mitat A. Birkan

Program Manager

Directorate of Aerospace and Engineering Sciences

Department of the Air Force

Bolling Air Force Base, DC 20332-6448

Prof. Michael Booty

Department of Mathematics

New Jersey Institute of Technology

Newark, NJ 07102-1982

Prof. John D. Buckmaster

Department of Aeronautical and Astronautical Engineering

University of Illinois

Urbana, II 61801

Prof. Sebastien Candel

Ecole Central des Arts et Manufactures

Grande Voie de Vignes

92290 Chatenay-Malabry

FRANCE

Dr. John Card

Department of Mechanical Engineering

Yale University

New Haven, CT 06520

Prof. J. F. Clarke

College of Aeronautics

Cranfield Institute of Technology

Cranfield-Bedford MK43 OAL

\section{ENGLAND}

Prof. Paul Clavin

Laboratoire Dynamique et Thermophysique des Fluides

Universite de Provence

Centre Saint Jerome

13397 Marseille Cedex 4

FRANCE

Prof. F. E. C. Culick

Jet Propulsion Center

California Institute of Technology

Pasadena, CA 91125 
Prof. Martin Golubitsky

Department of Mathematics

University of Houston

University Park

Houston, TX 77004

Prof. Michael Gorman

Department of Physics

University of Houston

Houston, TX 77004

Dr. Daryl D. Holm

CNLS, MS 457

Los Alamos National Laboratory

Los Alamos, NM 87545

Prof. G. M. Homsy

Department of Chemical Engineering

Stanford University

Stanford, CA 94305

Dr. G. Joulin

Laboratoirie D'Energetique et de Detonique

Universite de Poitiers

Rue Guillaume VII

86034 Poitiers

FRANCE

Dr. Hans Kaper

Applied Mathematics Division

Argonne National Laboratory

9700 S. Cass Ave.

Argonne, IL 60439

Prof. A. K. Kapila

Department of Mathematical Sciences

Rensselaer Polytechnic Institute

Troy, NY 12128

Prof. D. R. Kassoy

Department of Mechanical Engineering

University of Colorado

Boulder, CO 80309

Prof. Joseph B. Keller

Department of Mathematics

Stanford University

Stanford, CA 94305

Prof. Barbara Keyfitz

Department of Mathematics

University of Houston

University Park

Houston, TX 77004 
Prof. C. K. Law

Department of Mechanical and Aerospace Engineering

Engineering Quadrangle

Princeton University

Princeton, NJ 08544

Dr. Gary Leaf

Applied Mathematics Division

Argonne National Laboratory

9700 S. Cass Avenue

Argonne, IL 60439

Prof. Amable Liñán

Universidad Politecnica de Madrid

Escuela Tecnica Superior de Ingenieros Aeronauticos

Plaza del Cardenal Cisneros, 3

Madrid - 3

SPAIN

Prof. J. T. C. Liu

Division of Engineering, Box D

Brown University

Providence, RI 02912

Prof. Moshe Matalon

Department of Engineering Sciences and Applied Mathematics

Northwestern University

Evanston, II 60208

Prof. Bernard J. Matkowsky

Department of Engineering Sciences and Applied Mathematics

Northwestern University

Evanston, IL 60208

Prof. A. C. McIntosh

Department of Fuel and Energy

University of Leeds

Leeds LS2 9JT

United Kingdom

Prof. D. O. Olagunju

Department of Mathematical Sciences

University of Delaware

Newark, DE 19716

Prof. R. E. O'Malley

Department of Applied Mathematics

University of Washington Seattle, WA 98195

Prof. Norbert Peters

Institute fur Allgemeine Mechanik

Technische Hochschule Aachen

Aachen

GERMANY 
Prof. Victor Roytburd

Department of Mathematical Sciences

Rensselaer Polytechnic Institute

Troy, NY 12128

Prof. W. A. Sirignano

Office of the Dean

School of Engineering

University of California, Irvine

Irvine, CA 92717

Prof. L. Sirovich

Division of Applied Mathematics, Box F

Brown University

Providence, RI 02912

Prof. G. I. Sivashinsky

Department of Mathematics

Tel-Aviv University

Ramat-Aviv, Tel-Aviv 69978

ISRAEL

Prof. Mitchell D. Smooke

Department of Mechanical Engineering

Yale University

New Haven, CT 06520

Prof. D. Scott Stewart

Department of Theoretical and Applied Mechanics

University of minois

Urbana, IL 61801

Prof. C. H. Su

Division of Applied Mathematics, Box F

Brown University

Providence, RI 02912

Prof. Cesar Treviño

Departmento de Termica y Fluidos

Universidad Nacional Autonoma de Mexico

Facultad de Ingenieria

Patios No. 12, Jardines del Sur

MEXICO 23, D.F.

Prof. Vladimir Volpert

Department of Engineering Sciences and Applied Mathematics

Northwestern University

Evanston, IL 60208

Dr. David Weaver

Air Force Rocket Propulsion Laboratory

DYP/Stop 24

Edwards Air Force Base, CA 93523 
Prof. Forman A. Williams

Department of Applied Mechanics and Engineering Sciences

University of California, San Diego

La Jolla, CA 92093

Prof. Vigor Yang

Department of Mechanical Engineering

Pennsylvania State University

University Park, PA 16802

Prof. Benn Zinn

Department of Aerospace Engineering

Georgia Institute of Technology

225 North Avenue, NW

Atlanta, GA 30332

C. K. Westbrook, LLNL, L-321

MS 1110 R. C. Allen, 1422

MS 0834 A. C. Ratzel, 9112

MS 0834 M. R. Baer, 9112

MS 0834 M. L. Hobbs, 9112

MS 0834 R. J. Gross, 9112

MS 9001 T. O. Hunter, 8000

MS 9405 R. E. Stoltz, 8008

MS 9004 M. E. John, 8100

MS 9213 S. C. Johnston, 8103

MS 9054 W. J. McLean, 8300

MS 9163 W. Bauer, 8302

MS 9042 C. M. Hartwig, 8345

MS 9056 L. A. Rahn, 8351

MS 9051 W. T. Ashurst, 8351

MS 9051 A. R. Kerstein, 8351

MS 9052 D. R. Hardesty, 8361

MS 9055 R. Behrens, 8361

MS 9052 S. B. Margolis, 8361 (30)

MS 9053 R. W. Carling, 8362

MS 9021 Technical Communications Department, 8815, for OSTI (2)

MS 9021 Technical Communications Department, 8815/Technical Library, MS 0899, 4916

MS 0899 Technical Library, 4414 (4)

MS 9018 Central Technical Files, 8950-2 (3) 